\title{
Enterovirus antibody levels during the first two years of life in prediabetic autoantibody-positive children
}

\author{
K.Sadeharju ${ }^{1}$, M. Lönnrot ${ }^{1}$, T.Kimpimäki ${ }^{2}$, K.Savola ${ }^{3}$, S. Erkkilä ${ }^{4}$, T. Kalliokoski ${ }^{4}$, P.Savolainen ${ }^{4}$, P. Koskela ${ }^{5}$, \\ J. Ilonen ${ }^{6}$, O.Simell ${ }^{4}$, M. Knip ${ }^{7}$, H.Hyöty ${ }^{1}$
}

JDFI Center for Diabetes Prevention in Finland including:

${ }^{1}$ Department of Virology, University of Tampere and Tampere University Hospital, Tampere, Finland

${ }^{2}$ Department of Paediatrics, Tampere University Hospital, Tampere, Finland

${ }^{3}$ Department of Paediatrics, University of Oulu, Oulu, Finland

${ }^{4}$ Department of Paediatrics, University of Turku, Turku, Finland

${ }^{5}$ National Public Health Institute, Oulu, Finland

${ }^{6}$ Department of Virology, University of Turku, Turku, Finland

${ }^{7}$ Hospital for Children and Adolecents, University of Helsinki, Helsinki, Finland

\section{Abstract}

Aims/hypothesis. We evaluated the role of enterovirus infections in the pathogenesis of Type I (insulindependent) diabetes mellitus by monitoring enterovirus antibody levels in prediabetic children who turned positive for diabetes-associated autoantibodies in a prospective birth cohort study.

Methods. Serial serum samples taken during prospective observation starting at birth were analysed for IgG and IgA class antibodies against enterovirus antigens including purified coxsackievirus B4, echovirus 11, poliovirus 1 and a synthetic enterovirus peptide antigen using enzyme immunoassay. Maternal samples taken at the end of the third month of pregnancy were also studied. Analyses were done from 21 childen who developed autoantibodies and from 104 autoantibody-negative control children who were matched for the time of birth, gender and HLA susceptibility alleles. For comparison, adenovirus antibodies were also analysed from all samples collected. Results. IgG class enterovirus antibody levels were high in maternal samples and in cord blood in both case and control children. After birth the IgG levels decreased reaching a nadir at the age of 6 months. No IgA class antibodies were detected at birth but started to emerge postnatally. Antibody levels did not differ between the autoantibody positive and the control children during the first 6 months of life. From 6 months to 24 months of age, the autoantibody positive children had higher IgG and IgA levels against coxsackievirus B4, echovirus 11 and the synthetic enterovirus peptide antigens than control children but poliovirus 1 and adenovirus antibodies were closely similar in the two groups. The difference between children with autoantibodies and control children was predominantly seen among boys and among those with the HLA-DQB $1 * 0302 / x$ genotype. Conclusions/interpretation. Our data show that children who seroconverted for diabetes-associated autoantibodies develop stronger humoral immune responses to coxsackievirus B4, echovirus 11 and a synthetic enterovirus peptide antigen than children who remained negative for autoantibodies. Poliovirus antibodies induced by uniform vaccinations did not differ between the prediabetic and control children suggesting that the regulation of antibody responses to enteroviruses is not disturbed. Accordingly, the results imply a stronger enterovirus exposure in prediabetic children supporting the role of enteroviruses in the pathogenesis of Type I diabetes. [Diabetologia (2001) 44: 818-823]

Keywords Type I diabetes, enterovirus, antibody level, autoantibodies.
Received: 12 February 2001 and in revised form: 30 March 2001

Corresponding author: Dr. Heikki Hyöty, University of Tampere, Medical School, Department of Virology, PO Box 607, FIN-33101 Tampere, Finland, E-mail: blhehy@uta.fi

Abbreviations: CAV16, Coxsackievirus A16; CBV3, coxsackievirus B3; CBV4, coxsackievirus B4; DIPP, Diabetes Pre- diction and Prevention Study; EV11, echovirus 11; EIA, enzyme immunoassay; EIU, enzyme immunoassay unit; GADA, glutamic acid decarboxylase antibodies; IA-2A, protein tyrosine phosphatase IA-2 antibodies; IAA, insulin autoantibodies; ICA, islet cell antibodies; JDFU, Juvenile Diabetes Foundation Units; RU, relative unit. 
Type I (insulin-dependent) diabetes mellitus is caused by progressive immune-mediated destruction of pancreatic beta cells leading to insulin deficiency and hyperglycaemia [1]. The multifactorial pathogenesis comprises genetic and environmental components. Enterovirus infections are among the most suspected environmental triggers of the process. They are common human pathogens causing mainly mild respiratory symptoms or subclinical infections, although more severe manifestations, such as meningitis and myopericarditis, also occur. First enterovirus infections usually occur in early infancy but they are common at all ages [2].

Previous studies show that enterovirus antibody levels are higher in patients with newly diagnosed Type I diabetes than in healthy control children [3-6] and that enterovirus RNA is detected more frequently in the blood and serum of patients with Type I diabetes than in healthy control subjects [7-10]. Prospective studies have also shown an increased frequency of serologically confirmed enterovirus infections [11-14] and enterovirus RNA in serum of children who later turned positive for diabetes associated autoantibodies or developed clinical Type I diabetes $[14,15]$. Some studies have further suggested that maternal enterovirus infection during pregnancy could increase the risk for Type I diabetes in the offspring $[11,16]$. Accordingly, enteroviruses could initiate or accelerate the beta-cell damaging processes years before the manifestation of clinical Type I diabetes, perhaps already in utero.

We have now studied the role of enterovirus infections by analysing the development of enterovirus antibody levels in prospectively observed children who turned positive for diabetes associated autoantibodies compared with their matched control children who remained autoantibody-negative.

\section{Subjects and methods}

Subjects. The participants were from the Type I Diabetes Prediction and Prevention (DIPP) Study, which was initiated in Finland in 1994. In this study all babies born at the university hospitals in Turku, Oulu and Tampere are screened for HLADQB1 alleles that associate with increased risk for or protection from Type I diabetes as described [17].

The infants carrying the HLA-DQB $1 * 02 / * 0302$ or the $* 0302 / x$ genotype (x referring to alleles other than $* 02, * 0301$ or *0602) are then observed from birth with an interval of 3 to 6 months over the first 2 years of life and subsequently with an interval of 6 to 12 months. The follow-up samples are studied for islet cell antibodies (ICA). All samples from children who turn positive for ICA are then also analysed for autoantibodies against glutamic acid decarboxylase (GADA), insulin (IAA) and tyrosine phosphatase-related IA-2 protein (IA2A).

Those 21 children (10 boys), who were identified as the first ones in the DIPP cohort continuously positive for diabetes-associated autoantibodies were the cases in this study. The frequency of enterovirus infections, based on diagnostic increases in antibody levels and presence of enterovirus RNA in serum, and their correlation to the appearance of autoantibodies has been described [14]. Thus far, eight of them have progressed to Type I diabetes. The last follow-up sample from the remaining children was positive for ICA in 13/13 (mean level 54.5 JDFU), for IAA in 11/13 (mean level 1.51 RU), GADA in 10/13 (mean level 77.3 RU) and IA-2A in 4/13 (mean level 16.7 $\mathrm{RU}$ ). The last follow-up samples from these 13 children were thus positive for four autoantibodies in four children, for three autoantibodies in six, for one in two children (both have now been constantly ICA positive for at least 2 years) and in two autoantibodies in one child.

The islet autoantibody-positive children were born between November 1994 and June 1997 and have been followed from birth. The mean follow-up time was 20 months (range 9 to 24 months). Samples were drawn at birth (cord blood) and subsequently at 3 to 6-month intervals. Altogether 20 cord blood samples and 123 follow-up serum samples from the case children were available. Nine case children carried the high risk HLA-DQB $1 * 02 / * 0302$ genotype (three boys) and 12 cases had the moderate risk HLA-DQB $1 * 0302 / x$ genotype (seven boys).

Three to six (mean five) control children, matched for the time of birth, sex and the HLA-DQB1 alleles, were chosen from the DIPP cohort for each case. The control group comprised 104 children from whom altogether 98 cord blood samples and 562 follow-up serum samples were available. The control children were observed from birth according to the same protocol as the case children. All control children remained constantly negative for ICA.

In addition to the children's samples we also studied serum samples obtained at the end of the first trimester of pregnancy from the mothers of 20 islet autoantibody positive children and 103 control children.

Virus antibody analyses. IgG and IgA class antibodies against purified coxackievirus B4 (CBV4), echovirus 11 (EV11), poliovirus 1 , adenovirus hexon protein and a synthetic enterovirus peptide antigen (sequence KEVPALTAVETGAT-C derived from an immunodominant region of the capsid protein VP1 [17], which is a common epitope for several enteroviruses [19]) were measured using EIA as described [11, 20]. The purified CBV4 and EV11 were incubated at $+56^{\circ} \mathrm{C}$ for $15 \mathrm{~min}$ to expose antigenic determinants common for various enterovirus serotypes.

Microtitre plates (Nunc Immunoplate, Nunc, Glostrup, Denmark) were coated with the antigen at $1.0 \mu \mathrm{g} / \mathrm{ml}$ (CBV4, EV11, Polio1), $1.8 \mu \mathrm{g} / \mathrm{ml}$ (Adeno) on $2.5 \mu \mathrm{g} / \mathrm{ml}$ (bovine serum albumin-conjugated peptide) concentrations in carbonate buffer ( $\mathrm{pH} 9.4)$. Serum samples were analysed in 1/100 (IgA), $1 / 500$ (EV11 IgG) and 1/2000 (other IgG assays) dilution in PBS supplemented with $1 \%$ bovine serum albumin and $0.05 \%$ Tween 20 . Binding of antibodies was documented using peroxidase-conjugated anti-human IgG and IgA (P214 and P216, respectively, Dako, Copenhagen, Denmark).

All serum samples of a single child were analysed in the same assay. Results of the EIA tests were expressed in optical density values (OD 492).

Autoantibody analyses. Autoantibodies against islet cells (ICA), glutamic acid decarboxylase (GADA) and the protein tyrosine phosphatase-related IA-2 molecule (IA-2A) were analysed [21, 22]. Insulin autoantibodies (IAA) were measured with a recently described microassay [23]. The detection limit of ICA was 2.5 Juvenile Diabetes Foundation units (JDFU). The cut-off limits for positivity for IAA, GADA and IA$2 \mathrm{~A}$ were set at the $99^{\text {th }}$ centile in more than 370 non-diabetic 

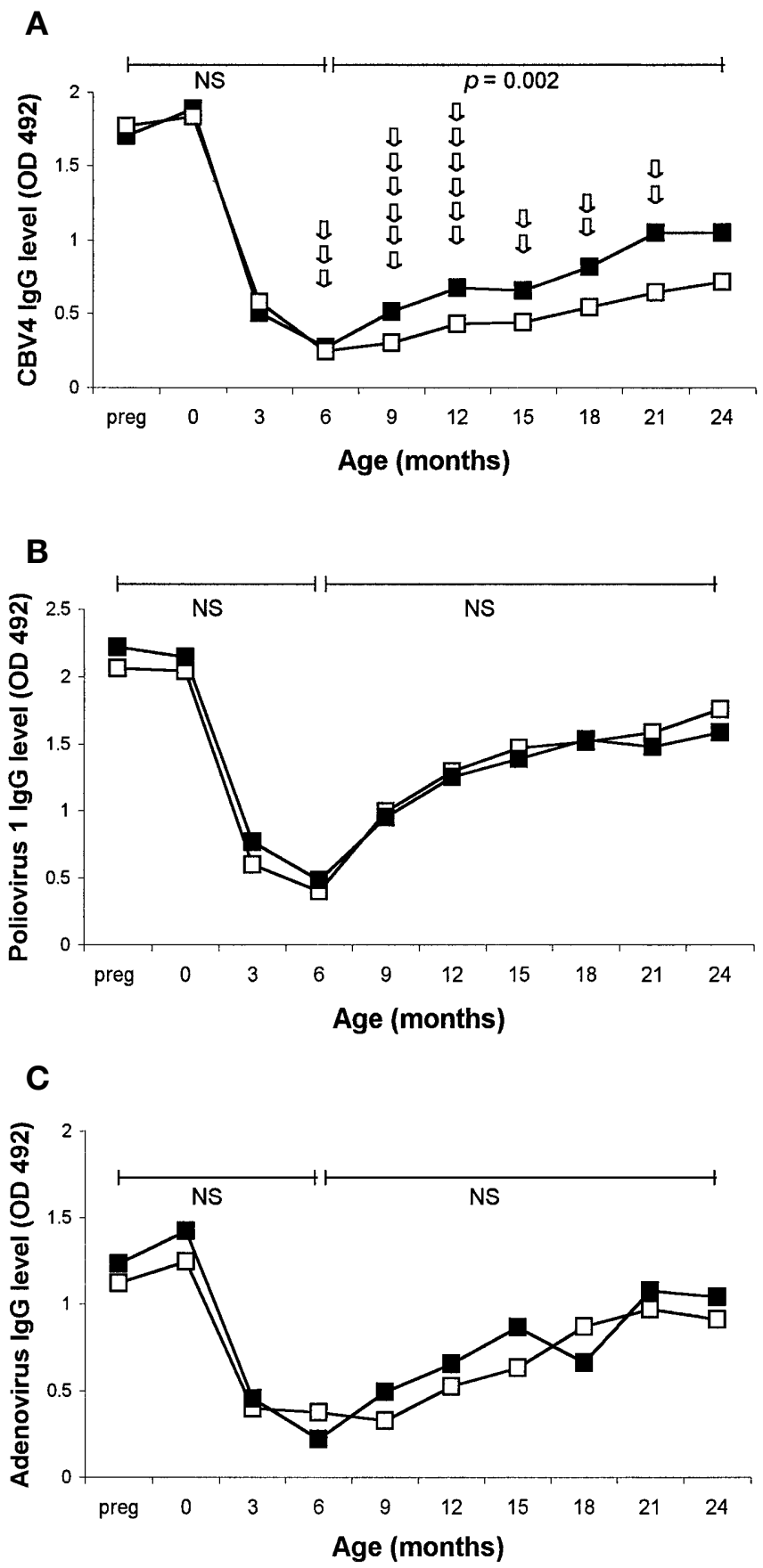

Fig.1 A-C. (A) Mean level of coxsackievirus B4 IgG antibodies in the follow-up sera of islet autoantibody positive and control children ( $\square$ islet autoantibody positive children, $\square$ control children). The age when autoantibodies were first discovered is marked by an arrow for each of the 21 case children. $P$ values were calculated using the area under the curve -method. (B) Mean level of poliovirus $1 \mathrm{IgG}$ antibodies in the followup sera of islet autoantibody positive and control children ( $\square$ islet autoantibody positive children, $\square$ control children). (C) Mean level of adenovirus IgG antibodies in the follow-up sera of islet autoantibody positive and control children ( $\square$ islet autoantibody positive children, $\square$ control children)
Finnish children (1.56 RU, 5.35 RU and 0.43 RU, respectively). All samples resulting in an autoantibody titre concentration between the 97.5 and 99.5 centiles were reanalysed to confirm autoantibody negativity or positivity.

Statistical analyses. Analyses based on the area under the curve-method was used in all comparisons [24]. The area under the antibody titre curve of each islet autoantibody positive child was compared with the mean area under the antibody titre curve of the matched control children. The follow-up periods of the islet autoantibody positive children and the control children were identical. Analyses were made from two periods: from pregnancy to the age of 6 months and from the age of 6 to 24 months. In IgG antibodies the first period represents mostly maternal antibodies and the second infant's de novo synthesized IgG antibodies. A $p$ value of less than 0.05 was considered statistically significant.

\section{Results}

The average IgG class enterovirus antibody levels were high in maternal samples and in cord blood samples of the islet autoantibody positive children and the control children. After birth IgG antibody levels decreased and reached a nadir at the age of 6 months. After that age the IgG levels started to increase. IgA antibodies were not detected in cord blood in any of these children but their levels started to increase postnatally.

During pregnancy and the first 6 months of life the islet autoantibody positive and the control children had comparable enterovirus and adenovirus antibody levels. Between 6 and 24 months of age the islet autoantibody positive children had higher enterovirus antibody levels than the control children. According to the area under the curve-analysis this difference was significant for CBV4-IgG $(p=0.002$, Fig. 1A), CBV4-IgA $(p=0.05)$, EV11-IgG $(p=0.03)$, EV11IgA $(p=0.005)$ and peptide-IgG $(p=0.03)$. Poliovirus or adenovirus antibody levels did not differ between the islet autoantibody positive children and the control children (Fig. 1B and C).

The difference between the islet autoantibody positive children and the control children was predominantly seen among boys (Fig. 2). They had higher levels of CBV4-IgG $(p=0.004)$, CBV4-IgA $(p=0.04), \quad$ EV11-IgG $\quad(p=0.008), \quad$ EV11-IgA $(p=0.02)$ and peptide-IgA $(p=0.003)$ than male control subjects.

All children in this cohort carried either the highrisk HLA-DQB1*02/*0302 genotype or the moderate-risk HLA-DQB $1 * 0302 / x$ risk genotype. The islet autoantibody positive children had higher enterovirus antibody levels than the control subjects in both HLA-DQB1 groups but the difference was larger when children with the HLA-DQB $1 * 0302 / x$ genotype were studied. Among those with the HLADQB $1 * 02 / * 0302$ genotype the difference between the islet autoantibody positive children and the con- 

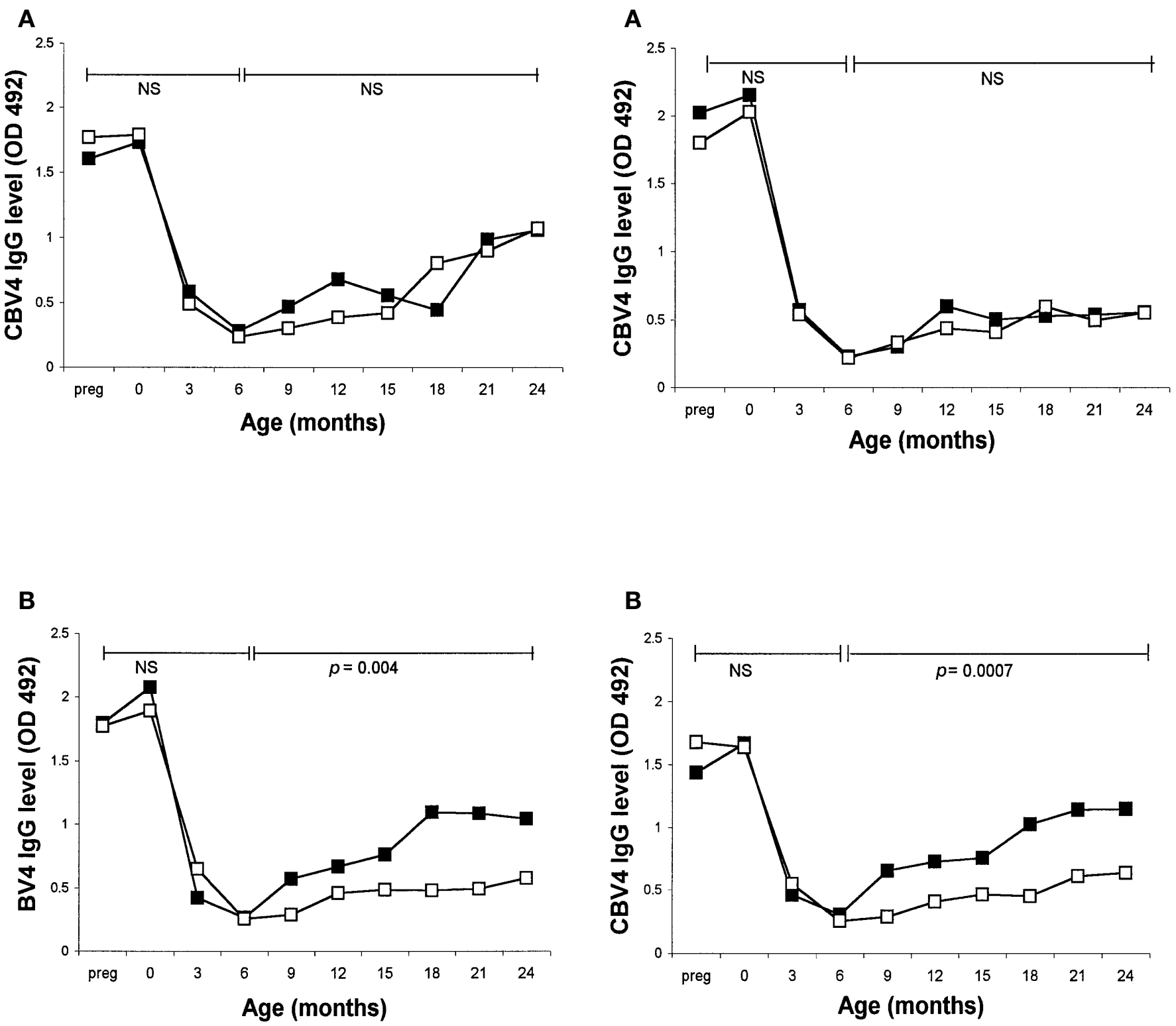

Fig. 2A, B. Mean level of coxsackievirus B4 IgG antibodies in the follow-up sera of islet autoantibody positive girls and controls (A) and islet autoantibody positive boys and their control (B) ( $\square$ islet autoantibody positive children, $\square$ control children)

trol children was significant for EV11- $\operatorname{IgG}(p=0.02)$, EV11- $\operatorname{IgA}(p=0.008)$ and peptide-IgA $(p=0.009)$, whereas in the HLA-DQB1*0302/x group the difference between the islet autoantibody positive and the control children was significant for all measured non-polio enterovirus antibody levels (Fig.3): CBV4-IgG $\quad(p=0.0007), \quad$ CBV4-IgA $\quad(p=0.004)$, EV11-IgG $(p=0.007)$, EV11-IgA $(p=0.008)$, peptide- $\operatorname{IgG}(p=0.04)$ and peptide- $\operatorname{IgA}(p=0.002)$.

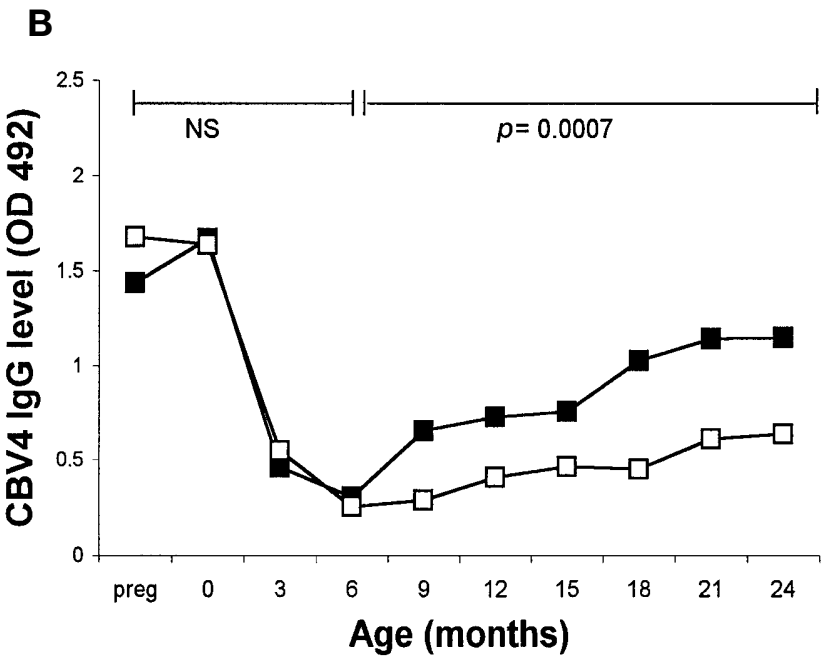

Fig. 3A, B. Mean level of coxsackievirus B4 IgG antibodies in the follow-up sera of children with the high-risk HLADQB $1 * 02 / * 0302$ genotype (A) and children with the moderate-risk HLA-DQB1*0302/x genotype (B) ( $\boldsymbol{\square}$ islet autoantibody positive children, $\square$ control children)

\section{Discussion}

Maternal enterovirus and adenovirus IgG antibody levels were high reflecting the fact that these infections are common. Cord blood samples contained no IgA antibodies, whereas IgG antibody levels were comparable to those in the maternal sample because of the transplacental transport of maternal $\mathrm{IgG}$ class antibodies. During the first 6 months of life this maternally acquired IgG decreased steeply to be replaced by the infant's de novo synthetised IgG. Also IgA antibody levels started to increase postnatally. 
After the age of six months the autoantibody positive children had constantly higher IgG and IgA levels against CBV4, E11 and enterovirus peptide antigen as compared with the control children. This can be due to either stronger exposure to enterovirus antigens, that is a higher number of enterovirus infections, or abnormally strong humoral immune responsiveness to enterovirus antigens among the islet autoantibody positive children. The latter alternative was evaluated by measuring antibody responses evoked by standardized enterovirus exposure, namely poliovirus vaccine, among the case and the control children. Finnish children are vaccinated by inactivated poliovirus vaccine at the age of 6,12 and 20 to 24 months and the coverage of these vaccinations is very high, over $95 \%$. The lack of difference in poliovirus antibodies between autoantibody positive and negative subjects suggests that the regulation of antibody reponses to enterovirus antigens is not disturbed in children with signs of preclinical Type I diabetes. Thus, the higher levels of CBV4 and E11 antibodies in autoantibody positive subjects suggest an increased number of enterovirus infections rather than abnormal immune responsiveness in these children. However, the case children did not seem to be more susceptible to virus infections in general: they had equal levels of adenovirus antibody level as the control children suggesting equal exposure to adenoviruses in the islet autoantibody positive children and the control children. Our findings therefore imply that enterovirus infections are risk factors for beta-cell autoimmunity.

In this study we used the area under the curve method to analyse the differences in antibody levels between the islet autoantibody positive children and the control children. This approach is optimal for this type of serial measurement [24], as it considers all the information obtained from serial measurements. It might also help to increase the sensitivity of prospective studies evaluating the frequency of virus infections particularly if the sample intervals are long and of variable duration. In addition to the area under the curve - analyses we compared antibody levels in islet autoantibody positive and control children at individual time-points using paired $t$ test (data not shown). Such analysis gave statistically significant differences only for CBV4 IgA antibody levels at the age of 12 and 21 months and for Peptide IgA antibody levels at the age of 12 and 18 months showing the higher sensitivity of the area under the curve-method in the analyses of data obtained at serial time points.

We have previously studied the number of enterovirus infections in these children by calculating the number of diagnostic increases in enterovirus antibody levels between subsequent serum samples and the number of samples positive for enterovirus RNA [14]. Enterovirus infections were found to be more frequent in the islet autoantibody positive children than in the control children and infections were also clustered close to the appearance of autoantibodies. Our present study is in line with our previous observations further supporting the role of enterovirus infections in the pathogenesis of Type I diabetes.

Complications of enterovirus infections such as meningitis or myocarditis, are more frequent among boys, although it is not known whether enterovirus infections are more common in boys than girls [25]. We reported in the Childhood Diabetes in Finland (DiMe) study that boys had a tendency towards seconversion to ICA positivity after enterovirus infections more often than girls [12]. In our present study the islet autoantibody positive boys had higher levels of enterovirus antibodies compared with the control children whereas no such difference was observed among the islet autoantibody positive girls and the control children, supporting the hypothesis that boys could be more susceptible to enterovirus-induced beta-cell damage.

The immune response to enteroviruses seems to be influenced by HLA-related factors. For example, the mean T-cell proliferation responses to enterovirus antigens are increased in subjects with the HLA-DR4 phenotype and decreased in subjects with the HLADR3 phenotype [26, 27]. In another study enterovirus specific IgM was particularly frequent in HLA-DR3 positive subjects with newly diagnosed Type I diabetes [28]. In our study the observed difference in the enterovirus antibody levels between the islet autoantibody positive and control children is not likely due to an effect of the HLA alleles because the case and control children were also matched for the HLA-DQB1 genotype. The difference in enterovirus antibodies between autoantibody positive and negative children was more pronounced among children with the HLADQB1*0302/x genotype (as a difference was seen in all measured enterovirus antibodies except poliovirus antibodies) than in children with the HLA-B $1 * 02 /$ *0302 genotype (in whom a difference was observed only in EV11-IgG, EV11-IgA and peptide-IgA). This finding suggests that children with the HLADQB $1 * 0302 / x$ genotype could be particularly susceptible to enterovirus-induced beta-cell damage.

Human enteroviruses include over 60 serotypes. In earlier studies particularly coxsackievirus B4 and B5 have been associated with Type I diabetes but also other serotypes could be diabetogenic [29-31]. We found that the case children had higher antibody levels than the control children against all tested antigens. A clear difference was observed in CBV4-IgG, but based on our data it is not possible to conclude that $\mathrm{CB}$ viruses are particularly diabetogenic, as only a few serotypes were included in our test panel and antibodies measured using this kind of EIA are not serotype specific. 
In conclusion, this study shows that children who developed persistent signs of beta-cell autoimmunity during prospective observation for the first two years of life had higher enterovirus antibody levels than children who remained autoantibody negative. This finding correlates with our previous observations suggesting that the frequency of enterovirus infections is increased in prediabetic children and supports the hypothesis that enterovirus infections have an important role in the pathogenesis of Type I diabetes.

Acknowledgements. This work was supported by the Juvenile Diabetes Research Foundation International (grants No. 395019 and No. 197114 to H.Hyöty, grant No. 197032 to M. Knip and grant No. 4-1998-274 to O. Simell), the Academy of Finland, the Sigrid Juselius Foundation, the Medical Research Foundation, the Diabetes Research Foundation, Finland, the Novo Nordisk Foundation, Foundation for Paediatric Research, Finland, the Finnish Virus Foundation and Jalmari ja Rauha Ahokas/Vuorisalo Foundation and the Medical Research Funds of Tampere, Oulu and Turku University Hospitals. We gratefully acknowledge the technical assistance of E. Jokela, M. Patrikainen, A. Karjalainen, I. Lehtimäki, A. Suominen, S. Valorinta, P. Salmijärvi, T. Mehtälä, S. Heikkilä and R. Päkkilä and thank the personnel working in the DIPP project. Special thanks go to the children and families who participate in the trial.

\section{References}

1. Atkinson MA, Maclaren NK (1994) The pathogenesis of insulin-dependent diabetes mellitus. N Engl J Med 331: 1428-1436

2. Juhela S, Hyöty H, Lönnrot M, Roivainen M, Simell O, Ilonen J (1998) Enterovirus infections and enterovirus specific T-cell responses in infancy. J Med Virol 54: 226-232

3. Gamble DR, Kinsley ML, Fitzgerald MG, Bolton R, Taylor KW (1969) Viral antibodies in diabetes mellitus. BMJ 3: 627-630

4. Barrett-Connor E (1985) Is insulin-dependent diabetes mellitus caused by coxsackievirus B infection? A review of the epidemiologic evidence. Rev Infect Dis 7: 207-215

5. Banatvala JE (1987) Insulin-dependent (juvenile-onset, type 1) diabetes mellitus coxsackie B viruses revisited. Prog Med Virol 34: $33-54$

6. Frisk G, Friman G, Tuvemo T, Fohlman J, Diderholm H (1992) Coxsackie B virus IgM in children at onset of Type I (insulindependent) diabetes mellitus: evidence for IgM induction by a recent or current infection. Diabetologia 35: 249-253

7. Clements GB, Galbraith DN, Taylor KW (1995) Coxsackie B virus infection and onset of childhood diabetes. Lancet 346: 221-223

8. Andréoletti L, Hober D, Hober-Vandenberghe C et al. (1997) Detection of coxsackie B virus RNA sequences in whole blood samples from adult patients at the onset of type 1 diabetes mellitus. J Med Virol 52: 121-127

9. Nairn C, Galbraith DN, Taylor KW, Clements GB (1999) Enterovirus variants in the serum of children at the onset of type 1 diabetes mellitus. Diabet Med 16: 509-513

10. Chehadeh W, Weill J, Vantyghem M-C et al. (2000) Increased level of interferon- $\alpha$ in blood of patients with insulin-dependent diabetes mellitus: relatinship with coxsackievirus B infection. J Infect Dis 181: 1929-1939

11. Hyöty H, Hiltunen M, Knip M et al. (1995) A prospective study of the role of Coxsackie B and other enterovirus infections in the pathogenesis of IDDM. Diabetes 44: 652-657
12. Hiltunen M, Hyöty H, Knip M et al. (1997) Islet cell antibody seroconversion in children is temporally associated with enterovirus infections. J Infect Dis; 175: 554-560

13. Lönnrot M, Knip M, Roivainen M et al. (1998) Onset of type 1 diabetes in infancy after enterovirus infections. Diabet Med 15: 431-434

14. Lönnrot M, Korpela K, Knip M et al. (2000) Enterovirus infection as a risk factor for beta-cell autoimmunity in the prospective birth-cohort trial DIPP. Diabetes 49: 1314-1318

15. Lönnrot M, Salminen K, Knip M et al. (2000) Enterovirus RNA in serum is a risk for beta-cell autoimmunity and clinical type 1 diabetes: a prospective study. J Med Virol 61: 214-220

16. Dahlquist GG, Ivarsson S, Lindberg B, Forsgren M (1995) Maternal enterovirus during pregnancy as a risk factor for childhood IDDM. A population-based case-control study. Diabetes 44: 408-413

17. Ilonen J, Reijonen H, Herva E et al. (1996) Rapid HLA-DQB1 genotyping for four alleles in the assessment of risk for IDDM in the Finnish population. Diabetes Care 19: 795-800

18. Roivainen M, Närvänen A, Korkolainen M, Huhtala ML, Hovi $\mathrm{T}$ (1991) Antigenic regions of poliovirus type 3/Sabin capsid proteins recognized by human sera in the peptide scanning technique. Virology 180: 99-107

19. Hovi T, Roivainen M (1993) Peptide antisera targeted to a conserved sequence in poliovirus capsid VP1 cross-react widely with members of the genus Enterovirus. J Clin Microbiol 31: 1083-1087

20. Samuelson A, Glimåker M, Skoog E, Cello J, Forsgren M (1993) Diagnosis of enteroviral meningitis with IgG-EIA using heat-treated virions and synthetic peptides as antigens. J Med Virol 40: 271-277

21. Savola K, Sabbah E, Kulmala P, Vähäsalo P, Ilonen J, Knip M (1998) Autoantibodies associated with Type I diabetes mellitus persist after diagnosis in children. Diabetologia 41: 1293-1297

22. Savola K, Bonifacio E, Sabbah E et al. (1998) IA-2 antibodies a sensitive marker of IDDM with clinical onset in childhood and adolescence. Diabetologia 41: 424-429

23. Williams AKJ, Bingley PJ, Bonifacio E, Palmer JP, Gale EAM (1997) A novel micro-assay for insulin antibodies. J Autoimmun 10: 473-478

24. Matthews JNS, Altman DG, Campbell MJ, Royston P (1990) Analysis of serial measurements in medical research. BMJ 300: 230-235

25. Morens D, Pallansch MA (1995) Epidemiology. In: Rotbart HA (ed.) Human Enterovirus Infections, ASM Press, Washington $\mathrm{p} 3-23$

26. Bruserud O, Stenerson M, Thorsby E (1985) T lymphocyte responses to coxsackie B4 and mumps virus. II. Immunoregulation by HLA-DR3 and -DR4 associated restriction elements. Tissue Antigens 26: 179-192

27. Bruserud O, Jervell J, Thorsby E (1985) HLA-DR3 and -DR4 control T-lymphocyte responses to mumps and coxsackie B4 virus: studies on patients with Type I (insulin-dependent) diabetes and healthy subjects. Diabetologia 28: 420-426

28. D'Alessio DJ (1992) A case-control study of group B coxsackievirus immunoglobulin $M$ antibody prevalence and HLA-DR antigens in newly diagnosed cases of insulin-dependent diabetes mellitus. Am J Epidemiol 135: 1331-1338

29. Frisk G, Nilsson E, Tuvemo T, Friman G, Diderholm H (1992) The possible role of Coxsackie A and echo viruses in the pathogenesis of type I diabetes mellitus studied by IgM analysis. J Infect Dis 24: 13-22

30. Helfand RF, Gary HE Jr, Freeman CY, Anderson LJ, Pallansch MA (1995) Serologic evidence of an association between enteroviruses and the onset of type 1 diabetes mellitus. Pittsburgh Diabetes Research Group. J Infect Dis 172: 1206-1211

31. Roivainen M, Knip M, Hyöty H et al. (1998) Several different enterovirus serotypes can be associated with prediabetic autoimmune episodes and onset of overt IDDM. J Med Virol 56: $74-78$ 